\title{
Results of a pilot study of patient-to-patient education strategy on self-management among glycemic uncontrolled patients with diabetes
}

This article was published in the following Dove Press journal:

Patient Preference and Adherence

13 April 2017

Number of times this article has been viewed

\section{Yingying Xiang \\ Pingyan Luo \\ Xueqin Cai \\ Yuqin Tang \\ Zhongming Wu}

Key Laboratory of Hormones and Development (Ministry of Health), Tianjin Key Laboratory of Metabolic Diseases, Tianjin Metabolic Diseases Hospital \& Tianjin Institute of Endocrinology, Tianjin Medical University, Tianjin, People's Republic of China
Correspondence: Zhongming Wu Tianjin Medical University Metabolic Diseases Hospital, No 66 Tongan Road, Heping District, Tianjin, 300070, People's Republic of China

Tel/fax +862223343729

Email wuzhongming@tmu.edu.cn
Objectives: The purpose of this study was to assess the efficacy and practicability of patient-to-patient (PTP) education strategy on glycemic control among patients with type 2 diabetes mellitus.

Methods: Eligible subjects from outpatient clinic were recruited and randomized to either intervention group or control group. Inpatients with poor outcomes and complications acted as mentors to share their experience with the intervention group in three tailored classes. Besides, the intervention group received general advice from specialists. The control group received general advice only. The glucose metabolic status and behavior modification indicators were evaluated before and after intervention. In addition, both groups finished a questionnaire survey regarding awareness to diabetic complications after intervention.

Results: Eighty-four subjects were recruited (42 subjects for each group), and 51 subjects finished the study. Both the intervention group $(n=29)$ and the control group $(n=22)$ showed a tendency toward a decrease in glycosylated hemoglobin level (A1c, $-0.8 \%$ vs $-0.4 \%$, $P<0.05)$ and improvement in behavior modification $(+5.0 \mathrm{vs}+2.8, P<0.05)$ after 6 months. The intervention group (13.8\%) obtained a higher percentage than the control group $(9.1 \%)$ whose A1c reached $\leq 7 \%$. The body mass index did not change significantly in any group. The questionnaire score about complication awareness was higher in the intervention group than in the control group.

Conclusion: This preliminary evidence suggests that PTP education strategy is acceptable for facilitating the outcome of glycemic control. Patient sense of complications may work on A1c reduction.

Keywords: patient-to-patient education strategy, diabetes, self-management, A1c

\section{Introduction}

Diabetes mellitus is a chronic and progressive disorder ${ }^{1,2}$ that requires continuing medical care and ongoing patient self-management education and support to prevent acute complications and reduce the risk of long-term complications. ${ }^{3-5}$ For adults with type 2 diabetes mellitus (T2DM), performing recommended self-care is essential for glycemic control; ${ }^{6-8}$ however, patient adherence remains challenging. ${ }^{9-11}$ In China, approximately two-thirds of patients with T2DM reported irregular self-monitoring of blood glucose. ${ }^{11}$ More endeavors are needed for a better T2DM management.

Transtheoretical model is widely known as stages of change theory according to which behavior changes can be divided into six stages as follows: pre-contemplation, contemplation, preparation, action, maintenance and termination. This influential model has been used in smoking cessation, vegetable and fruit intake, diabetes education 
and so on. ${ }^{12-14}$ Contemplation is an important step in which subjects learn the seriousness of behavioral problems, benefits from behavior change and also the cost for changes. At this stage, the intervention subject begins to produce the self-reevaluation of the behavior, weighs the pros and cons of behavior change and appears contradictory. But soon, the intervention subject will enter the next stage.

For current education strategies, most knowledge of diabetes is given by hospital staffs or educators, and patients learn the knowledge about self-management. However, less caution to the consequences of bad behavior and weak awareness to diabetic complications may make a part of patients slacken their efforts in behavior modification, especially for patients with longer duration of diabetes but no obvious body discomfort. There was tremendous power in speaking with someone in the same situation. ${ }^{15-19}$ Willis et al's study showed that patient-to-patient (PTP) interaction prior to seeing the physician in the waiting room has a positive effect on patient opinion of the pain clinic and the caregivers. ${ }^{20}$ In our study, the transtheoretical model was combined with peer education strategy, and the effect of PTP education strategy on self-management was evaluated.

\section{Methods}

\section{Study design}

A single-center, 6-month follow-up, randomized, controlled trial with two-group design was undertaken in the Metabolic Diseases Hospital in Tianjin (Figure 1). The study protocol was approved by the Tianjin Medical University Ethics Committee Review Board (No DXBYYhMEC2015-15) and was verified by the Chinese Clinical Trial Registry (No ChiCTR-IPR-15006366).

\section{Sample and setting}

Outpatients who met the following criteria were eligible to participate in the study: 1) aged between 30 and 70 years; 2) blood glucose was poorly controlled, glycosylated hemoglobin level (A1c) $\geq 7.5 \%$; 3 ) the duration of diabetes was $>3$ years and without obvious body discomfort (there were no distinct numbness of limbs, chest tightness and blurred vision; urinary albumin exclusion rate $\leq 300 \mathrm{mg} / 24$ hours) and 4) revisiting the same clinic specialist $>3$ months. Exclusion criteria included 1) type 1 diabetes or gestational diabetes; 2) any severe psychiatric disorders or comprehension barriers; 3 ) taking part in another

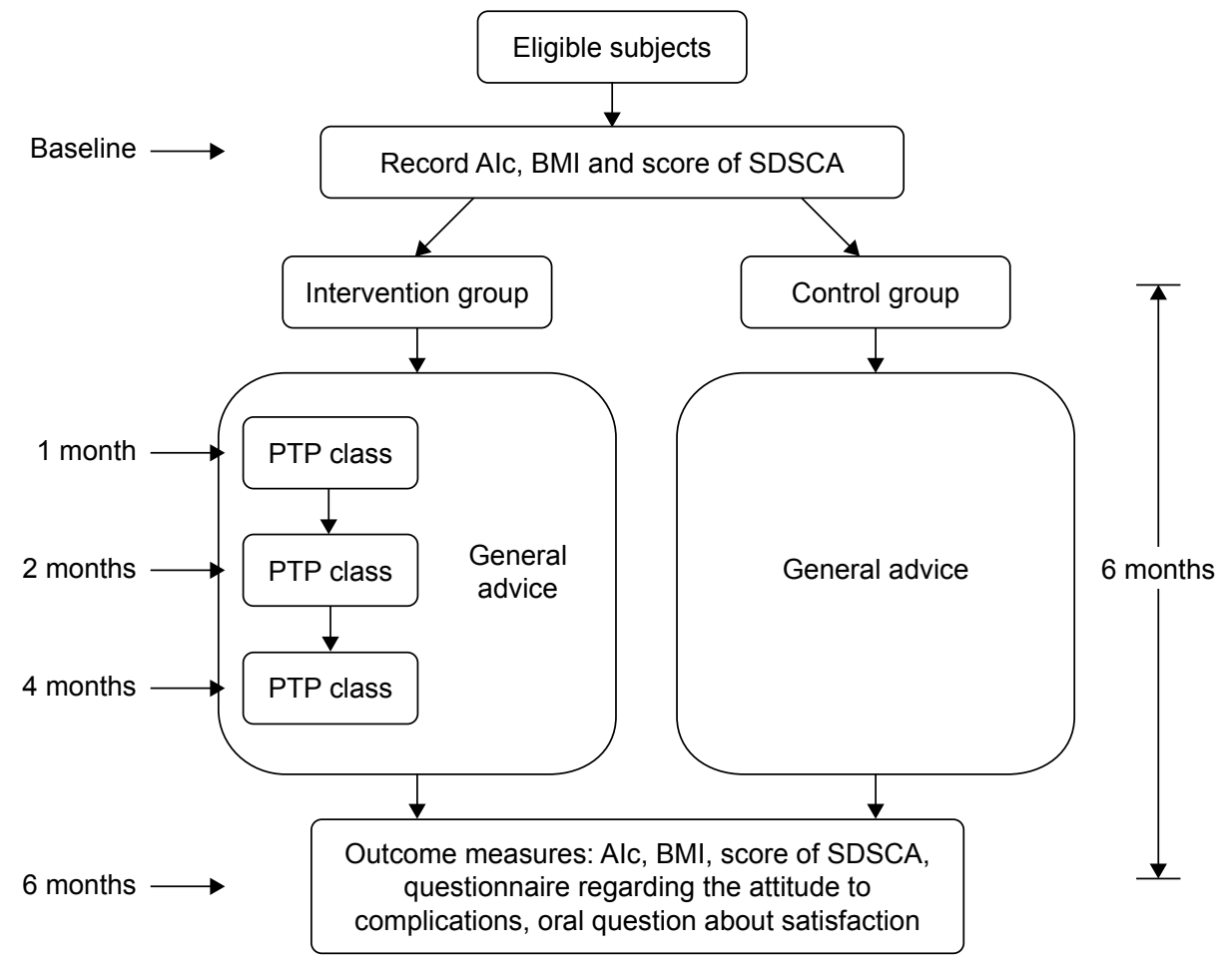

Figure I Flowchart of the 6-month follow-up study.

Notes: Intervention group was invited to attend PTP class and obtained general advice, twice in the first 3 months and once in the last 3 months. Control group obtained general advice only.

Abbreviations: Al c, glycosylated hemoglobin level; BMI, body mass index; PTP, patient-to-patient; SDSCA, summary of diabetes self-care activities. 
clinical trial; 4) history of trauma or surgery in recent 1 year; 5) with severe complications of diabetes mellitus and 6) alcohol or psychoactive substance abused.

Eligible subjects were explained the goals and risk of the study and gave their written informed consent at the beginning of the study. Subjects were randomized to the intervention or control group, which was carried out by an external agency using a computerized random number generator.

\section{Intervention}

\section{Intervention group}

Before the start of the study, subjects in the intervention group were told that they should attend a total of three PTP classes conducted at 1, 2 and 4 months, respectively. Besides that, they should keep visiting the clinic as their clinic physician advised. That is to say, the intervention subjects were gathered together for PTP class, and they visited the physician individually. In each PTP class, there were two parts of content. First, the subjects came to the education center and interacted with the inpatients who were selected from the ward by the resident doctor in advance. Apart from typical diabetic complications, the inpatients selected met the following criteria: $a>5$-year history of T2DM, blood glucose was stable after treatment and some personalities in common (eg, good interpersonal communication, easygoing, optimistic and would be willing to share their suffering with others). Inpatients were given short-time training after they agreed to take part in the PTP interaction. It was explained to the inpatients that the aim of the interaction was to share self-experience about diabetes and its complications with visitors but not the exact diabetic knowledge. PTP conversation topics were open while mostly related to 1) self-experience about the relationship between living habits and blood glucose level; 2) body discomfort caused by diabetic complications (eg, numbness of limbs, limp, foot ulcers, limited mobility, blurred vision, distress from dialysis); 3 ) experience with treatment (eg, economic burden, drug therapeutic effects and side effects) and 4) others (eg, depressed mood and loss of the quality of life). Fifteen inpatients were selected as volunteers, five volunteers in each class, five with diabetic nephropathy and undergoing hemodialysis for class one, five with typical diabetes foot for class two, and three with diabetic retinopathy and two with cardiopathy for class three; each class focused on one or two major diabetic complications. Under the guidance of hospital educators, the length of contact time lasted 1 hour.

Next, the educators made a short conclusion for 30 minutes concerning knowledge of the corresponding diabetes complication and the importance of glycemic control. For the intervention group, subjects who did not attend at least two of the three classes were excluded from this study.

\section{Control group}

The control group did not attend the class but was asked to keep visiting the clinic. Both the intervention group and the control group kept revisiting the same clinic specialist as before during the 6 months follow-up. General advice was delivered from clinic visit by specialists ${ }^{21}: 1$ ) healthy eating, 2) being active, 3) monitoring, 4) taking medications, 5) reducing risks and 6) problem discussion.

\section{Instruments}

Measurements were taken at baseline and the end of this trial; measurements included A1c, body mass index (BMI), summary of diabetes self-care activities (SDSCA) tool and a designed questionnaire. The SDSCA was developed by Toobert et al, ${ }^{22}$ which primarily contains 5 domains, dietary 4 items ( 1 item is reverse score), exercise 2 items, blood sugar monitoring 2 items, foot care 2 items and medicine taking 1 item; higher scores reflect higher degree of selfmanagement in that domain of care (each item score ranges from 1 to 7). Total SDSCA score is the sum of 5 domains and reflect the overall condition of self-management. Wang et $\mathrm{al}^{23}$ reported that SDSCA Chinese Version was practicable with acceptable Cronbach's $\alpha(0.82)$ and test-retest reliability (0.95). A questionnaire regarding the awareness and attitude to diabetic complications was designed by our team and was used by both groups after study. This questionnaire included three items and was based on 5 levels (from 0 to 4 points) to make quantification evaluation: 1) the susceptibility of getting diabetes complications under long-term hyperglycemia condition; 2) diabetes complication is a severity damage to human health and 3 ) treatment cost for diabetes complication is expensive. Scores range from 0 to 12 , and the higher the score is, the higher the crisis conscious is. In addition, there was an oral question about satisfaction to PTP contact after study. Patients could answer "Yes" or "No".

\section{Data analysis}

Data are represented as mean \pm standard deviation (SD) or frequency. Baseline and 6-month data were compared using a paired $t$-test, after normality of the data was confirmed using the Shapiro-Wilk test. When normality was not confirmed for some of the data sets (eg, baseline and 6-month blood glucose 
monitor in both groups; 6-month foot care in the intervention group and baseline foot care in the control group; baseline medication in the intervention group and 6-month medication in the control group), the Wilcoxon signed rank test was used. An independent $t$-test was used to compare the change in outcomes between the intervention and control groups. Again, normality of these data was confirmed using the Shapiro-Wilk test. For some of the data sets (change in blood glucose monitor, foot care and medication from baseline to post 6 months in the intervention group), normality was not confirmed, therefore the Mann-Whitney $U$-test. The statistical analyses were performed using SPSS windows version 13.0, and $P$-value $<0.05$ was considered for statistical significance.

\section{Results}

A total of 84 volunteers took part in this study at baseline, 42 in the intervention group and 42 in the control group. At the end of the study, $51(60.71 \%)$ subjects completed the 6-month follow-up, $29(69.05 \%)$ in the intervention group and 22 $(52.38 \%)$ in the control group. There were no statistical differences between the two groups in the basic characteristics of subjects who completed the follow-up (Table 1). Of the $39.28 \%(n=33)$ who withdrew, the main factors given were personal, including illness, lack of time, traveling and work-related issues, and there was no significant difference in comparison of baseline attributes between those who completed the study and those who did not.

Comparing the intervention group with the control group before and after intervention showed that there were no significant changes in BMI for either group (Table 2). Both groups had a significant reduction in A1c levels compared to their corresponding baseline record after 6 months. Notably, the intervention group had a reduction in A1c of $0.8 \%$ compared to their baseline value. At the end of the study, A1c levels were statistically significantly different between

Table I The basic characteristics of subjects who completed the study in both the intervention group and the control group

\begin{tabular}{lllll}
\hline Variables & $\begin{array}{l}\text { Intervention } \\
\text { group } \\
(\mathbf{n}=\mathbf{2 9 )}\end{array}$ & $\begin{array}{l}\text { Control } \\
\text { group } \\
(\mathbf{n}=\mathbf{2 2})\end{array}$ & $\mathbf{t} \chi^{2}$ & $P$-value \\
\hline Age (years) & $53.0 \pm 7.3$ & $55.4 \pm 9.7$ & 1.014 & 0.316 \\
$\begin{array}{l}\text { Disease duration (years) } \\
\text { Sex (male/female) }\end{array}$ & $7.1 \pm 2.7$ & $6.7 \pm 2.4$ & 0.577 & 0.567 \\
$\begin{array}{l}\text { Education (primary/ } \\
\text { middle/college) }\end{array}$ & $16 / 13$ & $1 \mathrm{I} / 1 \mathrm{I}$ & 0.134 & 0.714 \\
Hypertension (-/+) & $7 / 22$ & $9 / 10 / 3$ & 0.731 & 0.694 \\
\hline
\end{tabular}

Notes: The values are statistically significant at $P<0.05$. Data are presented as mean \pm SD or frequency.

Abbreviation: SD, standard deviation. the two groups. In the intervention group, the mean A1c decreased after 6 months from $8.7 \% \pm 1.3 \%$ to $7.9 \% \pm 0.9 \%$ $(P<0.001)$. In the control group, the mean A1c decreased from $8.7 \% \pm 0.9 \%$ to $8.3 \% \pm 0.8 \%(P<0.05)$. Data analysis revealed that PTP education combined with general advice played a role in lowering blood sugar $(-0.8 \%$ vs $-0.4 \%$, $P<0.05)$. A total of $13.8 \%$ subjects in the intervention group and $9.1 \%$ subjects in the control group reached $\leq 7 \%$ Alc level. For a more relaxed criterion of A1c, $51.7 \%$ subjects in the intervention group and $36.4 \%$ subjects in the control group reached $\leq 8 \%$ A 1 c level.

At the 6-month examination, the intervention group reported significantly more beneficial changes in the total SDSCA score $(+5.0 \mathrm{vs}+2.8, P<0.05)$ and observed a better improvement on their physical exercise $(+2.4 \mathrm{vs}+0.9$, $P<0.001)$ than the control group. Although there were no significant differences between the two groups at 6 months (intervention group vs control group), both groups showed a significant improvement in dietary behaviors from corresponding baseline to 6 months (intervention group +1.5 and control group +0.8 , respectively). Besides, the intervention group also showed a significant improvement in glucose monitoring $(+0.5, P<0.05)$. The foot care score did not change significantly in any group (Table 2).

Besides, the intervention group scored 10.97 \pm 0.94 (3.41, 3.69 and 3.86 corresponding to each item) from the questionnaire about awareness to diabetic complications and the control group scored 9.64 \pm 1.26 (2.86, 3.09 and 3.73 corresponding to each item). The total score of the intervention group was statistically higher than that of the control group $(P<0.001)$ (Table 3$)$. No safety concerns were identified during the study.

\section{Discussion}

The aim of diabetes care is to control blood glucose level and maintain body weight with effective strategies that are closely related to lifestyle improvement, including healthy diet as advised, regular exercise, adherence to diabetic medication and regular follow-up. ${ }^{24,25}$ In our study, PTP mentoring being done by really ill and out-of-control patients tended to have an impact on self-management and glycemic control. When comparing the results of the two groups, PTP strategy achieved a better reduction in A1c than that in the general advice group; higher percentage of subjects in PTP strategy met the A1c standard ( $\leq 7 \%$ or in relaxed criterion $\leq 8 \%$ ). A significant higher SDSCA score was achieved in the intervention group at the end of the study compared with the control group, reflecting the greater 
Table 2 Comparison of the clinical measures and behavior change between the two groups before and after intervention

\begin{tabular}{|c|c|c|c|c|c|c|c|}
\hline \multirow[t]{2}{*}{ Variables } & \multicolumn{2}{|c|}{ Intervention group $(n=29)$} & \multicolumn{2}{|c|}{ Control group $(n=22)$} & \multirow[t]{2}{*}{$P$-value ${ }^{a}$} & \multirow[t]{2}{*}{$P$-value ${ }^{b}$} & \multirow[t]{2}{*}{$P$-value } \\
\hline & Baseline & 6 months & Baseline & 6 months & & & \\
\hline Alc (\%) & $8.7 \pm 1.3$ & $7.9 \pm 0.9$ & $8.7 \pm 0.9$ & $8.3 \pm 0.8$ & $<0.001$ & 0.036 & 0.016 \\
\hline SDSCA & $23.7 \pm 3.1$ & $28.7 \pm 3.6$ & $23.5 \pm 3.9$ & $26.3 \pm 4.6$ & $<0.001$ & 0.002 & 0.025 \\
\hline Dietary & $9.7 \pm 1.9$ & $11.2 \pm 2.4$ & $10.1 \pm 2.7$ & $10.9 \pm 2.7$ & 0.001 & 0.044 & 0.249 \\
\hline Exercise & $3.5 \pm 1.5$ & $5.9 \pm 1.8$ & $3.3 \pm 1.7$ & $4.2 \pm 2.1$ & $<0.001$ & 0.005 & 0.001 \\
\hline Monitor & $3.0 \pm 1.2$ & $3.5 \pm 1.2$ & $2.8 \pm 0.9$ & $3.2 \pm 1.3$ & 0.027 & 0.070 & 0.556 \\
\hline Foot care & $2.7 \pm 1.2$ & $3.0 \pm 1.2$ & $2.6 \pm 0.9$ & $3.0 \pm 1.0$ & 0.198 & 0.216 & 0.731 \\
\hline Medication & $4.8 \pm 0.7$ & $5.0 \pm 1.2$ & $5.0 \pm 1.3$ & $5.0 \pm 1.0$ & 0.212 & 0.893 & 0.429 \\
\hline BMI $\left(\mathrm{kg} \cdot \mathrm{m}^{-2}\right)$ & $25.2 \pm 2.5$ & $25.0 \pm 2.3$ & $26.0 \pm 4.4$ & $25.7 \pm 4.2$ & 0.117 & 0.091 & 0.728 \\
\hline
\end{tabular}

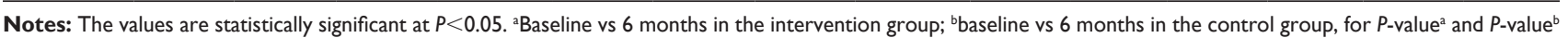
the results were analyzed with a paired $t$-test, Wilcoxon signed rank tests were used in monitor, foot care and medication; cintervention group vs control group (average change), data were assessed by an independent $t$-test, Mann-Whitney $U$-tests were used in monitor, foot care and medication. Data are presented as mean \pm SD. Abbreviations: Alc, glycosylated hemoglobin level; BMI, body mass index; SDSCA, summary of diabetes self-care activities.

mean improvement of behavior modification observed in the intervention group. Furthermore, the intervention group showed increased physical exercise and blood sugar monitoring score compared with baseline (Figure 2). Analysis of questionnaire about awareness to diabetic complications showed that both the intervention group and the control group got a relatively high score, suggesting that patients with long-term history of diabetes perceived some information about diabetic complications. However, the intervention group showed a much better improvement in A1c reduction and SDSCA score. This implies that diabetic complication awareness from others' suffering may move them forward to the next stage of behavior change. In practice, diabetes knowledge is delivered by hospital specialists seldom by experienced patients. Previous studies have demonstrated that peer education model had a positive effect on glucose control. ${ }^{16-18}$ Baksi et al ${ }^{18}$ showed that trained T2DM patients were as effective in imparting knowledge to their peers as specialist health professionals. In this study, inpatients with higher level of diabetes-related distress shared their sufferings and experience with the intervention group and increased the complication awareness in the intervention subjects. Even though the sample referred was small ( $n=51$ finally), the results of this study demonstrated that

Table 3 Score of questionnaire regarding awareness to diabetic complications

\begin{tabular}{llll}
\hline Variables & $\begin{array}{l}\text { Intervention } \\
\text { group }(\mathbf{n}=\mathbf{2 9})\end{array}$ & $\begin{array}{l}\text { Control } \\
\text { group }(\mathbf{n}=\mathbf{2 2})\end{array}$ & P-value $^{\mathbf{a}}$ \\
\hline Total score & $10.97 \pm 0.94$ & $9.64 \pm 1.26$ & $<0.001$ \\
Item I susceptibility & $3.41 \pm 0.63$ & $2.86 \pm 0.77$ & 0.010 \\
Item 2 severity & $3.69 \pm 0.54$ & $3.09 \pm 0.75$ & 0.002 \\
Item 3 cost & $3.86 \pm 0.35$ & $3.73 \pm 0.46$ & 0.238 \\
\hline
\end{tabular}

Notes: The values are statistically significant at $P<0.05$. antervention group vs control group, independent samples t-test were used in data analysis. Data are presented as mean \pm SD. there was a significant decrease in A1c levels and a better behavior change in the intervention group compared with the control population. All participants in the intervention group were satisfied with the PTP education model; they considered face-to-face interaction with inpatients to be beneficial and inspirited. From this novel education strategy, subjects learned about the harm of complications they might suffer directly from patients suffering those complications and not just by detached education by clinicians. PTP promoted the understanding of T2DM and its following complications comprehensively, gave force to glycemic self-management and improved the outcome of glycemic control among patients with uncontrolled blood glucose. In addition, it also works that subjects received diabetes knowledge from specialists, who offered assistance to solve the obstacles in behavior change and emotion regulation. At the same time, PTP interaction may magnify the effectiveness of general advice. Weight loss was not found in both groups, which confirms previous studies in Asian countries. ${ }^{26,27}$ The limitations of this study are the small sample size, single center and duration time. Despite all subjects had visit records in metabolic disease hospital before the study, subjects withdrawal in both groups is still a problem. Further refinement is needed for complication awareness measure because it has unknown psychometric properties.

\section{Conclusion}

The results of this study suggest that PTP education strategy is acceptable for facilitating the outcome of glycemic control. Patient complication awareness may influence the outcome of self-management. Future studies are needed to investigate whether the findings of this study are replicable in a similar setting and population, and, if so, to see if the findings can 

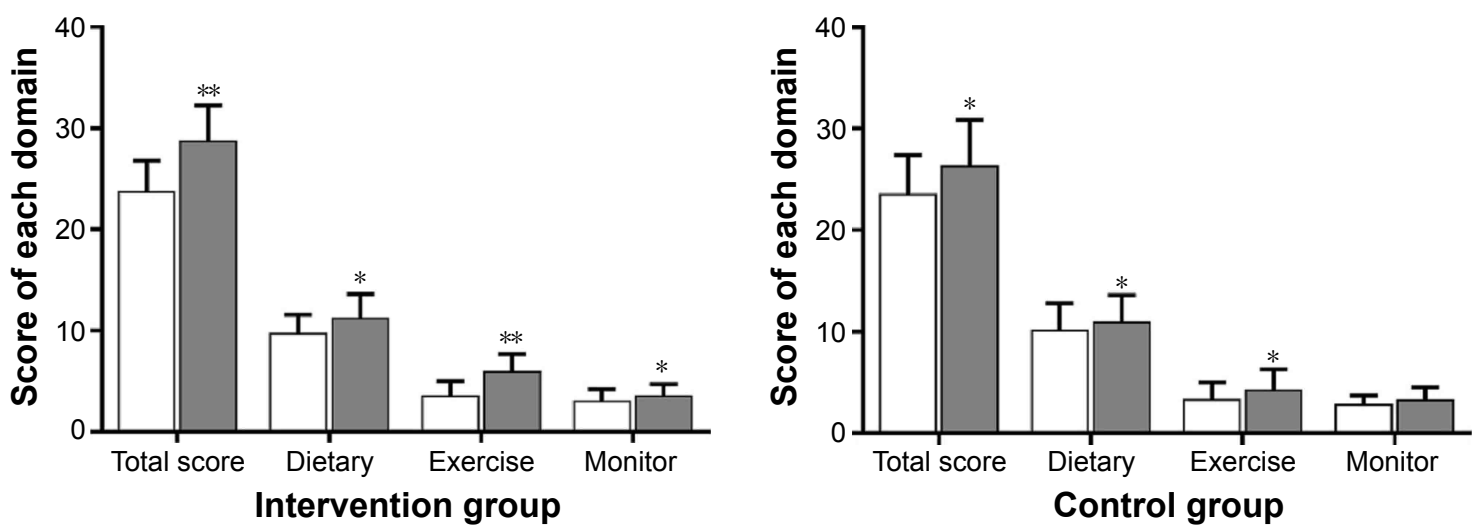

Baseline $\square 6$ months

Figure 2 SDSCA score of the two groups before and after intervention.

Notes: Total SDSCA score, dietary score, exercise score and blood sugar monitoring score were measured before and after intervention. Both groups had a significant improvement in total SDSCA score, dietary control and physical exercise compared to baseline; the intervention group also obtained a significant improvement in blood glucose monitoring. Baseline vs 6 months $\left({ }^{*}\right)$; $* P<0.05$, $* * P<0.001$.

Abbreviation: SDSCA, summary of diabetes self-care activities.

be generalized to other settings and populations, and whether it can be scaled up to a large number of people.

\section{Acknowledgment}

This work was supported by the National Natural Science Foundation of China (Grant 81671835).

\section{Disclosure}

The authors report no conflicts of interest in this work.

\section{References}

1. Whiting DR, Guariguata L, Weil C, Shaw J. IDF diabetes atlas: global estimates of the prevalence of diabetes for 2011 and 2030. Diabetes Res Clin Pract. 2011;94(3):311-321.

2. Guariguata L, Whiting DR, Hambleton I, Beagley J, Linnenkamp U, Shaw JE. Global estimates of diabetes prevalence for 2013 and projections for 2035. Diabetes Res Clin Pract. 2014;103(2):137-149.

3. Dudley B, Heiland B, Kohler-Rausch E, Kovic M. Education and technology used to improve the quality of life for people with diabetes mellitus type II. J Multidiscip Healthc. 2014;7:147-153.

4. Al-Khawaldeh OA, Al-Jaradeen N. Diabetes awareness and diabetes risk reduction behaviors among attendance of primary healthcare centers. Diabetes Metab Syndr. 2013;7(3):172-178.

5. Norris SL, Lau J, Smith SJ, Schmid CH, Engelgau MM. Self-management education for adults with type 2 diabetes: a meta-analysis of the effect on glycemic control. Diabetes Care. 2002;25(7):1159-1171.

6. American Diabetes Association. Standards of medical care in diabetes - 2013. Diabetes Care. 2013;36(suppl 1):S11-S66.

7. Loveman E, Frampton GK, Clegg AJ. The clinical effectiveness of diabetes education models for type 2 diabetes: a systematic review. Health Technol Assess. 2008;12(9):1-116.

8. Powers MA, Bardsley J, Cypress M, et al. Diabetes self-management education and support in type 2 diabetes: a joint position statement of the American Diabetes Association, the American Association of Diabetes Educators, and the Academy of Nutrition and Dietetics. Diabetes Educ. 2015;41(4):417-430.

9. Ho PM, Rumsfeld JS, Masoudi FA, et al. Effect of medication nonadherence on hospitalization and mortality among patients with diabetes mellitus. Arch Intern Med. 2006;166(17):1836-1841.
10. Saleh F, Mumu SJ, Ara F, Hafez MA, Ali L. Non-adherence to self-care practices \& medication and health related quality of life among patients with type 2 diabetes: a cross-sectional study. BMC Public Health. 2014; 14:431.

11. Ji L, Su Q, Feng B, et al. Glycemic control and self-monitoring of blood glucose in Chinese patients with type 2 diabetes on insulin: baseline results from the COMPASS study. Diabetes Res Clin Pract. 2016;112: $82-87$.

12. Koyun A, Eroğlu K. The effect of transtheoretical model-based individual counseling, training, and a 6-month follow-up on smoking cessation in adult women: a randomized controlled trial. Turk J Med Sci. 2016;46(1):105-111.

13. Hazavehei SM, Shahabadi S, Karami M, et al. The effective factors for fruit and vegetable consumption among adults: a need assessment study based on trans-theoretical model. Glob J Health Sci. 2016;8(10):203-211.

14. Salmela S, Poskiparta M, Kasila K, Vähäsarja K, Vanhala M. Transtheoretical model-based dietary interventions in primary care: a review of the evidence in diabetes. Health Educ Res. 2009;24(2):237-252.

15. Fisher EB, Boothroyd RI, Coufal MM, et al. Peer support for selfmanagement of diabetes improved outcomes in international settings. Health Aff (Millwood). 2012;31(1):130-139.

16. Dennis CL. Peer support within a health care context: a concept analysis. Int J Nurs Stud. 2003;40(3):321-332.

17. Elliott JA, Abdulhadi NN, Al-Maniri AA, Al-Shafaee MA, Wahlström R. Diabetes self-management and education of people living with diabetes: a survey in primary health care in Muscat Oman. PLoS One. 2013;8(2):e57400.

18. Baksi AK, Al-Mrayat M, Hogan D, Whittingstall E, Wilson P, Wex J. Peer advisers compared with specialist health professionals in delivering a training programme on self-management to people with diabetes: a randomized controlled trial. Diabet Med. 2008;25(9):1076-1082.

19. Dale J, Caramlau I, Sturt J, Friede T, Walker R. Telephone peerdelivered intervention for diabetes motivation and support: the telecare exploratory RCT. Patient Educ Couns. 2009;75(1):91-98.

20. Willis WK, Ozturk AO, Chandra A. The impact of patient-to-patient interaction in health facility waiting rooms on their perception of health professionals. Hosp Top. 2015;93(1):13-18.

21. Zheng YP, Wu LF, Su ZF, Zhou QH. Development of a diabetes education program based on modified AADE diabetes education curriculum. Int J Clin Exp Med. 2014;7(3):758-763.

22. Toobert DJ, Hampson SE, Glasgow RE. The summary of diabetes self-care activities measure: results from 7 studies and a revised scale. Diabetes Care. 2000;23(7):943-950. 
23. Wang JS, Wang RH, Ling CC. Self-care behaviors and related factors in outpatients newly diagnosed with noninsulin-dependent diabetes mellitus. J Nurs. 1998;45:60-75.

24. Jalilian F, Motlagh FZ, Solhi M, Gharibnavaz H. Effectiveness of selfmanagement promotion educational program among diabetic patients based on health belief model. J Educ Health Promot. 2014;3:14.

25. Brunisholz KD, Briot $P$, Hamilton $S$, et al. Diabetes self-management education improves quality of care and clinical outcomes determined by a diabetes bundle measure. J Multidiscip Healthc. 2014;7:533-542.
26. Kim HS, Choi W, Baek EK, et al. Efficacy of the smartphone-based glucose management application stratified by user satisfaction. Diabetes Metab J. 2014;38(3):204-210.

27. Guo HL, Wu ZM, Yu DM. Effectiveness of goal-setting on glycemic control in poor outpatients with diabetes. Diabetes Res Clin Pract. 2012; 96(2):e40-e42.

\section{Publish your work in this journal}

Patient Preference and Adherence is an international, peer-reviewed, open access journal that focuses on the growing importance of patient preference and adherence throughout the therapeutic continuum. Patient satisfaction, acceptability, quality of life, compliance, persistence and their role in developing new therapeutic modalities and compounds to optimize clinical outcomes for existing disease states are major areas of interest for the journal. This journal has been accepted for indexing on PubMed Central. The manuscript management system is completely online and includes a very quick and fair peer-review system, which is all easy to use. Visit http://www. dovepress.com/testimonials.php to read real quotes from published authors.

\footnotetext{
Submit your manuscript here: http://www.dovepress.com/patient-preference-and-adherence-journal
} 